\title{
A 30 ps timing resolution for single photons with multi-pixel Burle MCP-PMT
}

\author{
J. Va’vra*, J. Benitez, J. Coleman, D.W.G.S. Leith, G. Mazaheri, B. Ratcliff, \\ J. Schwiening \\ SLAC, Stanford University, CA 94309, U.S.A.*
}

\begin{abstract}
We have achieved 30 psec single-photoelectron and 12 ps for multi-photoelectron timing resolution with a new 64 pixel Burle MCP-PMT with 10 micron microchannel holes. We have also demonstrated that this detector works in a magnetic field of $15 \mathrm{kG}$, and achieved a single-photoelectron timing resolution of better than 60 psec. The study is relevant for a new focusing DIRC RICH detector for particle identification at future Colliders such as the super B-factory or ILC, and for future TOF techniques. This study shows that a highly pixilated MCP-PMT can deliver excellent timing ersolution.
\end{abstract}

\section{Introduction}

The DIRC detector for the BaBar experiment provides excellent particle identification performance $[1,2]$. We are developing a next generation DIRC, which is capable not only measuring an $x \& y$ coordinate of each photon with similar angular resolution to the present BaBar DIRC, but, in addition, each photon's time-of-propagation (TOP [3]) through the Fused Silica bar with $\leq 150$ ps timing resolution (the present BaBar DIRC has a timing resolution of only $\sigma \approx 1.6 \mathrm{~ns}$ ). Here we present studies of the limit of the timing resolution of a new 64-pixel Burle/Photonis MCP-PMT with $10 \mu \mathrm{m}$ holes. This tube has been shown to perform well in the magnetic field of $15 \mathrm{kG}$. One expects to achieve better timing

\footnotetext{
* Corresponding author. Tel.: 650-926-2658; fax: 650-926-4178; e-mail: jjv@slac.stanford.edu.

** Work supported by the Department of Energy, contract DEAC02-76SF00515.
} 
resolution because the tube is faster than the MCPPMT tubes with $25 \mu \mathrm{m}$ holes used in the present focusing DIRC prototype ${ }^{1}$. With such tube one could achieve better DIRC performance at shorter photon path lengths.

In addition to RICH applications, there is a new interest to push the TOF performance to new limits approaching $\sigma \approx 5$-10ps with new MCP-PMT type of detectors $[6,7,8]$.

\section{Photon detector and experimental setup}

Figure 1 shows the 64-pixel MCP-PMT 85012501 used in this study. The tube has $10 \mu \mathrm{m}$ holes, $6 \times 6 \mathrm{~mm}^{2}$ pixel size, and the cathode-to-MCP distance of $6-7 \mathrm{~mm}$, which means that the recoiling electrons can land up to $\sim 12 \mathrm{~mm}$ away from the original point of impact, and this in turn causes a long timing tail. The tail can be eliminated if the gap is reduced to $\sim 0.75 \mathrm{~mm}[4]$.

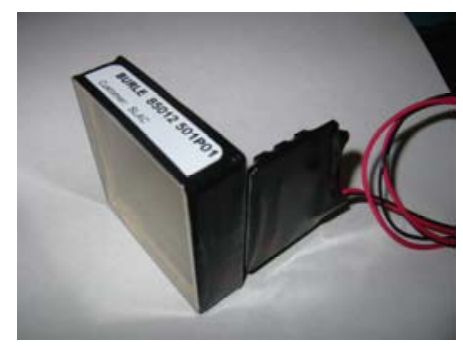

Fig. 1. Burle/Photonis “open area” 64-pixel MCP-PMT 85012501 (S/N 11180401) with $10 \mu \mathrm{m}$ hole diameter.

The timing resolution study was done on a single pad, illuminated at its center, and connected coaxially to a fast amplifier $~ 1 \mathrm{~ns}$ away, while all other pads were grounded; this represents an ideal case. This arrangement was fixed while varying amplifiers of various noise and bandwidth (BW) performance, coupled to two types of constant fraction discriminators (CFD), and LeCroy TDC with 24ps/count - see Table 1.

\footnotetext{
${ }^{1}$ We obtained $\sigma \sim 54 \mathrm{ps}$ in an ideal best case, $\sigma \sim 70-80 \mathrm{ps}$ in average case for Burle 85011 MCP-PMT with $25 \mu \mathrm{m}$ holes [4,5].
}

To do this measurement, we use the PiLas 635nm laser diode, ${ }^{2}$ for which the manufacturer quotes a timing resolution of $\sim 35 \mathrm{ps}$ FWHM for timing between an internal electrical trigger and a light pulse. The light was attenuated with optical filters and brought to a detector via a multi-mode $62.5 \mu \mathrm{m}$ dia. fiber, equipped with lenses at both ends to make a small sub-mm spot on the photocathode. For some measurements we tried to improve the timing resolution by triggering on light, which was achieved by an optical splitter and feeding one branch to a $2 \mathrm{GHz}$ bandwidth $\mathrm{Si}$ diode. No improvement was achieved compared to the PiLas electronic trigger.

\section{Single photoelectron timing resolution}

Figure 2 shows the best single photoelectron timing resolution results: $\sigma \approx 32 \pm 0.6 \mathrm{ps}$ with both Hamamatsu $1.5 \mathrm{GHz}$ BW and Ortec VT120A $\sim 0.4 \mathrm{GHz}$ BW amplifiers. The resolution was evaluated with a double-Gaussian fit function. Table 1 summarizes all results with other amplifier choices. As we pointed out in footnote 1 , the best ever single photoelectron timing resolution obtained with the MCP-PMT with $25 \mu \mathrm{m}$ holes was $\sigma \approx 54 \pm 4$ ps $[4,5]$.

The overall conclusion is that in the "singlephotoelectron domain," it is sufficient to have a relatively slow amplifier $(\sim 0.4 \mathrm{GHz} \mathrm{BW})$ to achieve a timing resolution of $\sigma \approx 32 \mathrm{ps}$, provided that its noise performance is good, and the amplifier is fast enough to follow fast pulses from the MCP-PMT. For example, a combination of two Elantek chips with an overall voltage gain of $130 \mathrm{x}$ with a rise time of $\sim 1.5 \mathrm{~ns}$ is too slow for this type of tube, althouh such speed is sufficient for MCP-PMT with $25 \mu \mathrm{m}$ hole diameter - see Table 1 for various examples.

For comparison, it is interesting to point out that the best result we have achieved in the same setup with a Geiger mode APD (G-APD or SiPMT) was $\sigma_{\text {narrow }} \approx 38$ ps and $\sigma_{\text {wide }} \approx 111$ ps [4]. This indicates that the new vacuum Burle MCP-PMT competes well with a G-APD performance.

\footnotetext{
${ }^{2}$ PiLas laser diode is made by Advanced Laser Diode Systems, D12489 Berlin, Germany.
} 

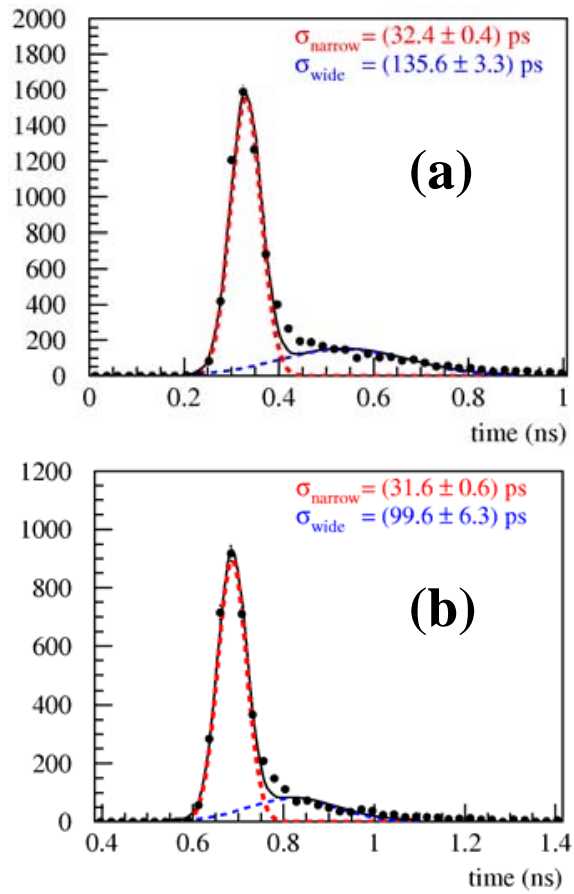

Fig. 2. Single photoelectron timing resolution in Burle 64-pixel MCP-PMT 85012-501 with $10 \mu \mathrm{m}$ hole diameter, and B = 0kG, using (a) Hamamatsu C5594-44 1.5GHz BW amplifier, and (b) Ortec VT120A $\sim 0.4$ GHz BW amplifier.

\section{Single photoelectron timing resolution at 15kG}

This particular measurement was performed with Burle 4-pixel MCP-PMT 85001-501 P01 with $10 \mu \mathrm{m}$ hole diameter (a different tube than the tube used for the rest of the measurement in this paper). The MCPPMT was placed in a dipole capable of reaching $\sim 15 \mathrm{kG}$. The magnetic field direction was nominally perpendicular to MCP face, however, this angle could be changed in small steps up to an angle of $15^{\circ}$. We have achieved the timing resolutions close to $\sigma \approx 50 \mathrm{ps}$ at $2.7 \mathrm{kV}(\sim 150 \mathrm{~V}$ lower than allowed maximum voltage, which means we have not reached the best possible limit of the resolution) - see Fig. 3. The measurement was made using the Ortec VT120A amplifier with a voltage gain of 200x (in this case we did not use a $6 \mathrm{~dB}$ att.). Larger amplifier gain was necessary in this case as there is a large MCP gain drop at higher magnetic field (more than a factor of five as one goes from $B=0 k G$ to $B=15 k G$ ).

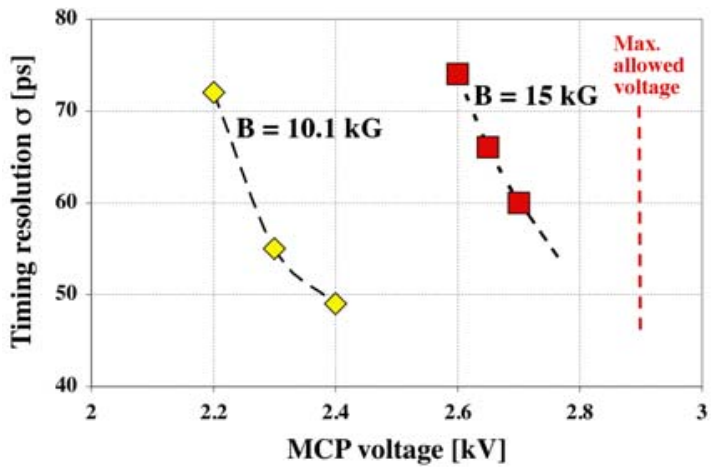

Fig. 3. Single photoelectron timing resolution using Ortec VT120A 0.4GHz BW amplifier connected to Burle 4-pixel MCPPMT 85001-501 P01 with $10 \mu \mathrm{m}$ hole diameter, and B = 10.1 \& 15 kG.

We also found that the MCP can be tilted by $3-5^{\circ}$ (angle between a normal to the MCP face relative to the field axis) with no effect on the pulse height. At $10^{\circ}$, one observes a factor of two in reduction of the pulse height, i.e., the tube can still be used. However, above $15^{\circ}$ angle, the pulse height is reduced by a factor of ten.

\section{Multi-photoelectron timing resolution}

The aim of this measurement was (a) to determine the electronics contribution to the measurements shown in Fig. 2, and (b) to investigate the limit of the MCP-PMT resolution for a larger number of photoelectrons, a region relevant to the TOF counters.

Fig. 4 shows that the limiting resolution is reached for Npe $>20$ photoelectrons, and is $\sigma \approx 13 \mathrm{ps}$ for Hamamatsu $1.5 \mathrm{GHz} \mathrm{BW}$, and $\sigma \approx 24 \mathrm{ps}$ for the Ortec VT120A $\sim 0.4 \mathrm{GHz}$ BW amplifiers. No matter how hard we tried, we could not reduce the 24ps resolution. We conclude that in the "multiphotoelectron domain" the speed of the amplifier is crucial. One is tempted to follow a simple explanation: the expected timing resolution with a CFD discriminator is $\sigma_{\mathrm{t}} \approx \sigma_{\mathrm{A}} /\left(\mathrm{ds}_{\mathrm{o}} / \mathrm{dt}\right)_{\mathrm{t}=0}$, where $\sigma_{\mathrm{A}}$ and $\left(\mathrm{ds}_{0} / \mathrm{dt}\right)_{\mathrm{t}=0}$ are the noise and the slope measured at the zero-crossing point. Based on these measurements we would then expect to obtain $\sigma_{\mathrm{t}} \approx 12 \mathrm{ps}$ for the Hamamatsu amplifier, and $\sigma_{\mathrm{t}} \approx 17 \mathrm{ps}$ for the Ortec 
VT120A amplifier. However, reality in the "10ps resolution domain" is more complicated as $\mathrm{t}$ resolution is fine tune of signal/noise ratio, detector response, amplifier and CFD bandwidths, amplifier pulse shape uniformity (partial saturation), CFD delay, CFD threshold, amplifier-to-CFD delay, TDC resolution, TDC diff. linearity, wiring impedance, thermal drifts, ground loops, etc.

Although the resolution in Fig. 4b is constant for Npe $>20$, there is a walk of the mean value even with the CFD discriminator, and therefore it is necessary to measure a pulse height (ADC), if Npe is varying in a real application. One is therefore tempted to conclude that a simple discriminator coupled to a TDC with an ADC off-line correction is sufficient to do a good timing in a practical application.

\section{Acknowledgments}

We would like to thank M. McCulloch for help to prepare various setups.

\section{References}

[1] I. Adam et al., "Particle Identification System for the BaBar experiment,” Nucl. Instr.\&Meth., A538(2005)281-357.

[2] B.N. Ratcliff, Nucl. Instr.\&Meth., A502(2003)211.

[3] Definition: $\operatorname{TOP}\left(\Phi, \theta_{\mathrm{c}}, \lambda\right)=\left[\mathrm{L} / \mathrm{v}_{\mathrm{g}}(\lambda)\right] \mathrm{k}_{\mathrm{z}}\left(\Phi, \theta_{\mathrm{c}}\right), \theta_{\mathrm{c}}$ Cherenkov angle, $\mathrm{L}$ - distance of light travels in the bar, $\mathrm{v}_{\mathrm{g}}(\lambda)$ - group velocity of light, $\lambda$ - photon wavelength, and $k_{z}\left(\Phi, \theta_{c}\right)$ - z-component of the unit velocity vector.

[4] C. Field, T. Hadig, D.W.G.S. Leith, G. Mazaheri, B. Ratcliff, J. Schwiening, J. Uher and J. Va'vra, "Development of photon detectors for a fast focusing DIRC,” Nucl. Instr.\&Meth., A553(2005)96-106.

Table 1

Single photoelectron timing resolution $=\mathrm{f}$ (amplifier bandwidth) for 64-pixel MCP-PMT with $10 \mu \mathrm{m}$ holes, at 2.80kV, and B = 0kG

\begin{tabular}{|c|c|c|c|c|c|c|}
\hline Amplifier type & $\begin{array}{c}\text { Bandwidth } \\
\text { [GHz] }\end{array}$ & $\begin{array}{c}\text { Total voltage } \\
\text { gain }\end{array}$ & $\begin{array}{c}\mathrm{V}_{\mathrm{pp}} \\
\text { Noise/Signal } \\
{[\mathrm{mV}]}\end{array}$ & CFD type & $\begin{array}{c}\text { Resolution } \\
\sigma_{\text {narrow }}, \sigma_{\text {wide }} \\
\text { [ps] }\end{array}$ & Comment \\
\hline Ortec VT120A + 6dB att. & $\sim 0.4$ & $100 x$ & $\sim 1 / 450$ & Phillips 715 & 32,100 & The best result \\
\hline Hamamatsu C5594-44 & 1.5 & $63 x$ & $1-2 / 450$ & Phillips 715 & 32,135 & Very good \\
\hline Ortec 9306 & 1.0 & $100 x$ & $\sim 8 / 400$ & Ortec 9307 & 43,134 & Worse S/N \\
\hline Tandem of 2 THS- $4303^{\mathrm{a}}$ & $\sim 0.5$ & $30-40 x$ & $\sim 8 / 200$ & Phillips 715 & 47,120 & Fast, but bad S/N \\
\hline Tandem of 2 Elantek-2075 & $\sim 0.2$ & $130 \mathrm{x}$ & $\sim 10 / 50$ & Phillips 715 & - & Slow, bad S/N \\
\hline
\end{tabular}

[5] C. Field, T. Hadig, M. Jain, D.W.G.S. Leith, G. Mazaheri, B. Ratcliff, J. Schwiening, and J. Va'vra, "Novel photon detectors for focusing DIRC prototype," Nucl. Instr.\&Meth., A518(2004)565-568.

[6] M. Akatsu et al., "MCP-PMT timing property for single photons,” Nucl. Instr.\&Meth., A528(2004)763-775.

[7] “10 ps workshop,” Arlington, TX, USA, March 28, 2006, organizer A. Brandt.

[8] T. Credo et al., "Picosecond TOF measurement for Colliders using Cherenkov Light,” IEEE Nucl. Sci. Conference Recorfs, Vol. 1, 16 Oct., 2004.
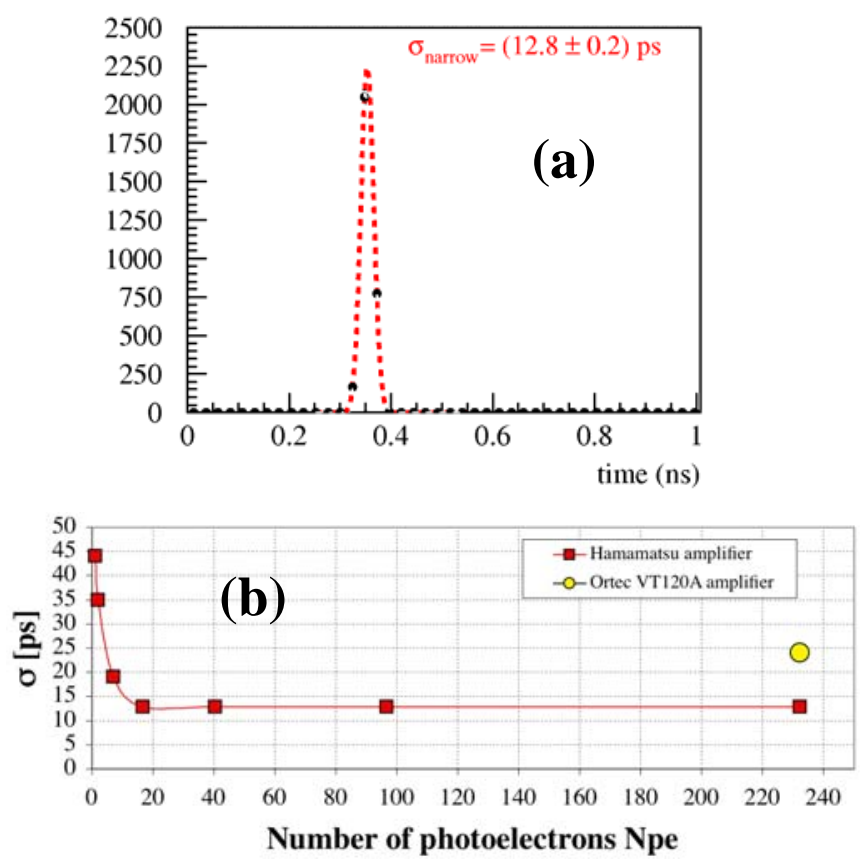

Fig. 4. Multi-photoelectron timing resolution using Ortec VT120A $\sim 0.4 \mathrm{GHz}$ BW amplifier connected to Burle 64-pixel MCP-PMT 85012-501 with 10 $\mu \mathrm{m}$ hole diameter, and B $=0 \mathrm{kG}$, for (a) Npe 232 , and (b) varying Npe.

${ }^{\mathrm{a}}$ THS-4303 and Elantek-2075 chips have a bandwidth of 1.5GHz for a gain of 10x. 